\title{
Integrated visceral leishmaniasis surveillance system in primary care for children in Meshkin-Shahr district, north-western Islamic Republic of Iran
}

M. Mohebali, Gh.H. Edrissian, ${ }^{7}$ M.R. Shirzadi, ${ }^{2}$ Gh. Hosseingholizadeh, ${ }^{3}$ M.H. Pashaei, ${ }^{3}$ A. Ganji, ${ }^{3}$ Z. Zarei, ${ }^{4}$ A. Kousha, ${ }^{5}$ B. Akhoundi, ${ }^{7}$ H. Hajjaran ${ }^{7}$ and H. Malekafzali ${ }^{6}$

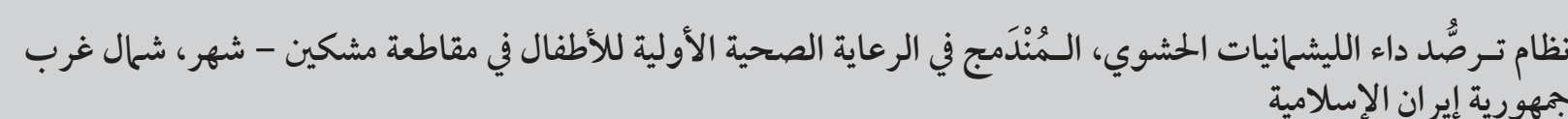

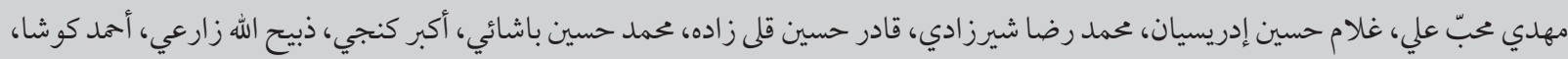

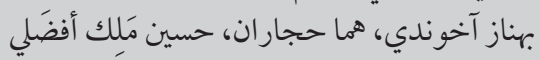

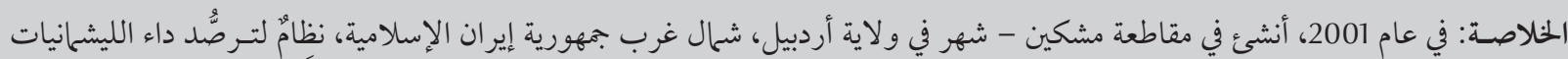

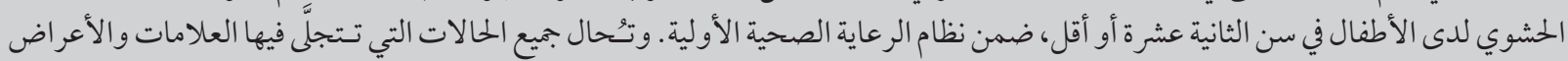

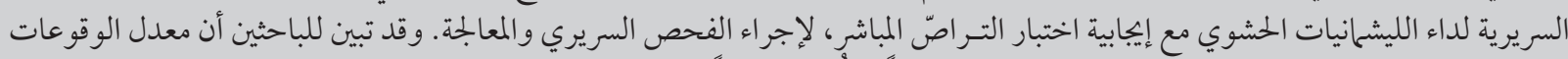

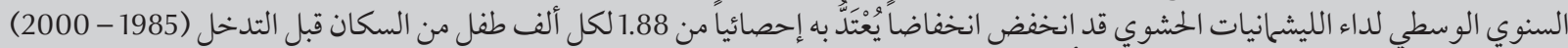

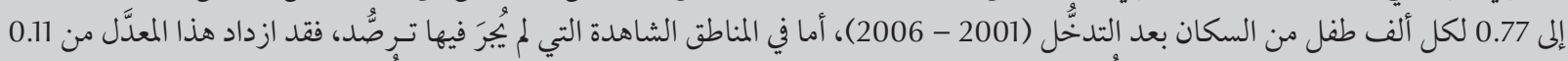

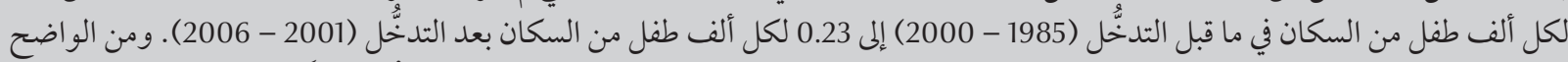

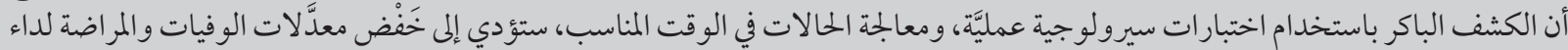

$$
\begin{aligned}
& \text { الليشمانيات الحشوي في المناطق الموطونة. }
\end{aligned}
$$

ABSTRACT In 2001 a visceral leishmaniasis (VL) surveillance system was set up for children aged $\leq 12$ years in the primary health system in Meshkin-Shahr district of Ardebil province, north-western Islamic Republic of Iran. All cases with clinical signs and symptoms of VL and positive by the direct agglutination test were referred for physical examination and treatment. The mean annual incidence of VL decreased significantly from 1.88 before (1985-2000) to 0.77 per 1000 child population after the intervention (2001-07). In a control area with no surveillance, it increased from 0.11 to 0.23 per 1000. Early detection of VL using practical serological tests and timely treatment of cases could decrease the mortality and morbidity rates of VL in endemic areas.

Système de surveillance intégrée de la leishmaniose viscérale dans les soins primaires pour les enfants dans le district de Meshkin-Shahr, nord-ouest de la République islamique d'Iran

RÉSUMÉ En 2001, un système de surveillance de la leishmaniose viscérale a été mis en place pour les enfants âgés de 0 à 12 ans dans le système de santé primaire du district de Meshkin-Shahr, province d'Ardebil, nord-ouest de la République islamique d'Iran. Tous les cas présentant des signes cliniques et des symptômes de leishmaniose viscérale ainsi qu'une réaction positive au test d'agglutination directe étaient orientés en vue d'un examen physique et d'un traitement. L'incidence annuelle moyenne de la leishmaniose viscérale a nettement diminué, passant de 1,88 avant l'intervention (1985-2000) à 0,77 pour 1000 enfants après l'intervention (2001-2006). Elle a augmenté dans une zone témoin sans surveillance, passant de 0,11 à 0,23 pour 1000 enfants. Un dépistage précoce de la leishmaniose viscérale à l'aide de tests sérologiques pratiques et une prise en charge rapide des cas permettraient de réduire les taux de mortalité et de morbidité de la leishmaniose viscérale dans les zones endémiques.

${ }^{7}$ Department of Medical Parasitology and Mycology; ${ }^{4}$ Meshkin-Shahr Research Station, National Institute of Health Research of Islamic Republic of Iran; ${ }^{6}$ Department of Epidemiology and Biostatistics, Tehran University of Medical Sciences, Islamic Republic of Iran (Correspondence to M. Mohebali: mohebali@tums.ac.ir).

${ }^{2}$ Zoonoses Control Unit, Diseases Management Centre, Ministry of Health, Treatment and Medical Education, Tehran, Islamic Republic of Iran. ${ }^{3}$ Meshkin-Shahr Health Centre, Ardebil, Islamic Republic of Iran.

${ }^{5}$ Tabriz University of Medical Sciences, East Azerbaijan, Islamic Republic of Iran.

Received: 26/06/08; accepted: 03/11/08 


\section{Introduction}

Visceral leishmaniasis (VL) — caused by Leishmania infantum LON-49 (GenBank accession no. EF653268) in human and animal reservoirs - has been reported from different parts of the Islamic Republic of Iran $[1,2]$. In recent decades, VL has become an important endemic disease among rural and seminomadic populations in the north-west of the country [1]. In a longitudinal, cross-sectional seroepidemiological study carried out during 1996-2006, a total of 32855 human serum samples were collected from 4 geographical zones of the Islamic Republic of Iran; 3574 samples $(10.9 \%)$ were positive at titres of $\geq 1: 3200$. Almost $43 \%$ of the samples were collected from MeshkinShahr and Moghan districts in Ardebil province in the north-west of the country [3], an area where health facilities are not well-established and VL often coexists with brucellosis, tuberculosis and other infectious diseases. Moreover, $99.4 \%$ of the VL cases were in children under 12 years of age.

A VL surveillance system for active and passive case-finding was set up in the villages of Meshkin-Shahr district in 2001 to carry out early detection in children aged under 12 years in the primary stage of infection using serological methods [4] and to treat infected individuals promptly to decrease the mortality and morbidity rates of the disease in endemic areas. In the present study, the incidence of VL in children in the intervention area was compared before and after the intervention and in a control area where the surveillance system was not established.

\section{Methods}

\section{Study design}

In this longitudinal study a VL surveillance system was established for children $\leq 12$ years old in Meshkin-Shahr district (intervention area) in 2001. The annual incidence of VL in children of this age group was compared over 2 periods, before (1985-2000) and after the intervention (2001-06). In order to control for ecological changes or changes in living facilities that might affect the VL incidence rate, an endemic area adjoining Meshkin-Shahr was selected where a VL surveillance system was not established, Ahar district (control area). The annual incidence of $\mathrm{VL}$ in this area was similarly compared over the period for which relevant data were available, both before (1992-2000) and after the intervention (2001-06) and with the incidence in the intervention area.

\section{Study area}

Meshkin-Shahr district is located in the north-west of the Islamic Republic of Iran. It covers an area of approximately $1530 \mathrm{~km}^{2}$ and its population is estimated to be 237585 , among whom $29.7 \%$ are settled in urban areas and $70.3 \%$ live in 323 rural areas. MeshkinShahr is an important endemic focus of VL in the Islamic Republic of Iran and several VL studies have been conducted in this area $[5,6]$. The control villages in Ahar district have similar ecology and socioeconomic characteristics to the Meshkin-Shahr villages.

Health services in the study areas are delivered through the district health network, consisting of a local hospital and a few health centres in the city, and health centres with health houses in rural areas. The health house (khaneh behdasht) is the first level of rural health services and covers several satellite villages with similar cultural and social characteristics to a range of about $1500 \mathrm{~m}$. One of the most important activities of health workers (behvarz) in the health houses is local disease control and data collection.

The second level is the rural health centre (RHC) situated in a village and covering a population up to 7500 . It is run by a medically trained doctor (usually a general physician). The main functions of the RHC are to provide backup services to rural health houses, offer diagnostic and treatment services to cases referred by the behvarz and refer those needing more specialist care to the district health centre (DHC) or district hospital.

The third level in the rural health network is the DHC, which is mainly concerned with the supervision and coordination of the activities of RHCs and liaison with more specialized district hospitals.

The data for this study were collected by trained behvarz in the health houses, trained technicians in the RHCs and medically trained physicians in the DHC and district hospitals.

\section{Sample collection}

There were around 100 behvarz during 2001-07 serving an average population of Meshkin-Shahr of 179000 with 29868 children aged $\leq 12$ years. In the control area there were 117 behvarz for an average population of 237585 and 23062 children $\leq 12$ years.

The behvarz of all health houses in Meshkin-Shahr district were trained to recognize and refer all suspected cases of VL to an RHC. Finger-prick blood samples were collected onto filter paper by the behvarz from children who were suspected of having VL, i.e. if they had all 3 of the clinical symptoms: abdominal distension, paleness and fever of at least 2 weeks duration [5,7]. In MeshkinShar districts where the number of VL cases was high, active case-finding and investigation of the animal reservoir hosts, particularly stray and owned dogs, were also carried out.

The blood samples were sent first to the RHC then to the local VL laboratory in the DHC where they were tested using the direct agglutination test (DAT) by trained technicians. Seropositive patients were referred to a paediatrician or general physician in the only hospital in Meshkin-Shahr city for further physical examination and treatment. Some children were referred to private or public physicians in Meshkin-Shahr. In some cases, bone marrow aspirates were also 
obtained for the detection of Leshmania spp. from patients with clinical signs and symptoms of VL but with no positive DAT results. All confirmed cases, i.e. those with positive DAT and clinical manifestations of VL (fever, paleness, anaemia, splenomegaly and hepatomegaly), were referred to the DHC to receive appropriate treatment. Pentavalent antimony in meglumine antimoniate (Glucantime, Rorer Rhone-Poulenc Specia, Paris, France) was administered intramuscularly at $20 \mathrm{mg} / \mathrm{kg}$ body weight daily for $21-28$ days.

Since, the VL surveillance system had not been set up in the control area, the incidence was estimated from clinical features, response to the treatment and occasionally DAT (only in symptomatic patients).

\section{DAT antigen preparation and serological tests}

DAT was developed as a simple, reliable, cost-effective tool for diagnosis and epidemiological surveys of VL in human and canine reservoirs in the Islamic Republic of Iran [3,4,8-10].

DAT Leishmania antigen was supplied by the Protozoology Unit of the Department of Medical Parasitology and Mycology at the School of Public Health, Tehran University of Medical Sciences $[3,10]$. All the collected serum samples were tested by DAT. Initially, for screening purposes, 2 dilutions of serum samples (1:800 and 1:3200) were tested with Leishmania antibodies. If both of these dilutions were positive, samples with titres 1:800 were diluted further to give endpoint titres of 1:102 400. Control wells with negative serum samples and known negative and positive controls were tested on each plate daily. The positive standard control was prepared from the serum of humans with $L$. infantum infection from the endemic areas, confirmed by microscopy, culture, animal inoculation and 1:102 400 DAT titres. A titre of $\geq 1: 3200$ was used as the cut-off point for human Leishmania infection, as determined in our previous studies $[1,3,10]$.

\section{Statistical analysis}

The mean annual incidence of VL per 1000 population aged $\leq 12$ years old based on positive DAT accompanied by clinical signs and symptoms and relative risk of the disease with 95\% confidence interval (CI) were calculated. Data were compared in the periods before and after establishment of surveillance in Meshkin-Shahr villages and with data from the control area. Analyses were done using the chi-squared, $t$-test and paired $t$-tests with SPSS software, version 13.5. $P$-value $<0.05$ was considered statistically significant.

\section{Results}

During 2001-06 a total of 3977 human serum samples were collected from children suspected of having VL in the intervention area. The seroprevalence rate at titres $\geq 1: 3200$ was $4.15 \%$.
Table 1 shows the incidence of $\mathrm{VL}$ in children $\leq 12$ years old, based on positive DAT and clinical signs and symptoms, in the intervention and control areas before and after the invention started in 2001. The mean annual incidence of VL decreased significantly from 1.88 per 1000 population aged $\leq 12$ years in the period before the intervention (1985-2000) [5] to 0.77 per 1000 after the intervention (2001-07) $(P<0.001)$. In the control area, the mean annual incidence of VL increased significantly from 0.11 per 1000 population aged $\leq 12$ years in the period before 2001 (1992-2000) [5] to 0.23 per 1000 in the period after the intervention (2001-07) $(P<0.005)$.

Figure 1 shows the mean annual incidence each year in the intervention and control areas for the period 2001-07, i.e. after the intervention.

During the intervention period, there were only 2 registered deaths due to $\mathrm{VL}$ in the intervention area (including an 8-year-old boy who did not tolerate meglumine antimoniate or amphotericin B), while at least 10 deaths caused by VL were registered in the intervention area before the surveillance intervention. The annual mortality rate due to VL in children up to 12 years in the intervention area decreased significantly from 0.16 per 1000 before to 0.009 per 1000 population aged $\leq 12$ years after the intervention $(P<0.001)$. About 20 deaths due to VL in children up to 15 years old were registered in the control villages during this period.

\begin{tabular}{|c|c|c|c|c|c|c|c|}
\hline \multirow[t]{2}{*}{ Study area } & \multicolumn{2}{|c|}{ Before intervention } & \multicolumn{2}{|c|}{ After intervention } & \multirow[t]{2}{*}{$\mathbf{R R}$} & \multirow[t]{2}{*}{$95 \% \mathrm{Cl}$} & \multirow[t]{2}{*}{$P$-value } \\
\hline & $\begin{array}{l}\text { VL cases } \\
\text { detected }^{\mathrm{a}} \\
\text { No. }\end{array}$ & $\begin{array}{l}\text { Mean annual } \\
\text { incidence } \\
\text { (/1000 children } \\
\leq 12 \text { years) }\end{array}$ & $\begin{array}{l}\text { VL cases } \\
\text { detected }^{\mathbf{b}} \\
\text { No. }\end{array}$ & $\begin{array}{l}\text { Mean annual } \\
\text { incidence } \\
\text { (/1000 children } \\
\leq 12 \text { years) }\end{array}$ & & & \\
\hline Intervention area & 1798 & 1.88 & 161 & 0.77 & 0.43 & $0.28-0.68$ & $<0.001$ \\
\hline Control area & 68 & 0.11 & 69 & 0.23 & 2.06 & $0.28-1.72$ & $<0.001$ \\
\hline
\end{tabular}

aPeriods of data collection: intervention area 1985-2000; control area 1992-2000.

${ }^{b}$ Periods of data collection: intervention area 2001-2007; control area 2001-2007.

$R R=$ relative risk; $\mathrm{Cl}=$ confidence interval . 


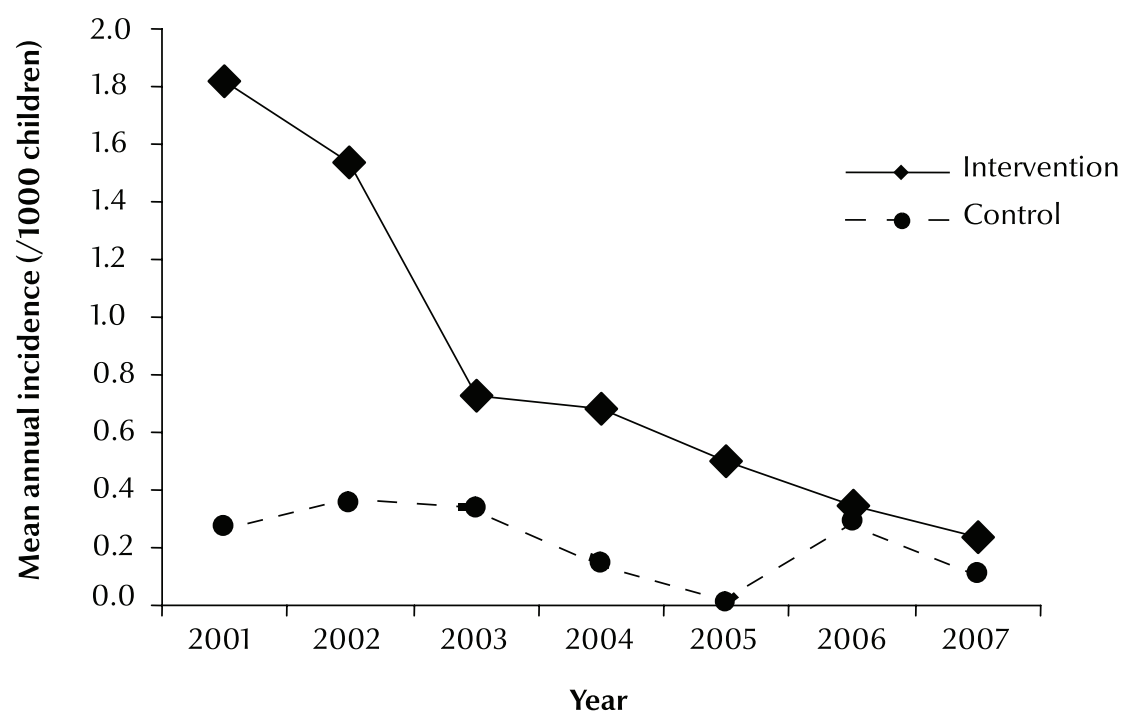

Figure 1 Mean annual incidence of visceral leishmaniasis (VL) per 1000 children aged $\leq 12$ years in the intervention area (Meshkin-Shahr district) and control area (Ahar district) after establishment of the VL surveillance system

\section{Discussion}

Control of zoonotic visceral leishmaniasis (ZVL) is difficult and the current control strategies for ZVL rely on reservoir and vector control, the use of insecticide-impregnated materials to prevent insect bites, and active case detection with appropriate treatment to avoid the spread of infection $[11,12]$. Despite the evidence from experimental studies of a decreased incidence of ZVL in both dogs and children following serological screening of dogs and killing of seropositive animals, the efficiency and acceptability of control strategies for ZVL are still debated $[13,14]$. Treating infected dogs is not an effective control strategy, as relapses are frequent and dogs can regain infection weeks after treatment, despite showing clinical cure [15]. Moreover the widespread veterinary use of $\mathrm{VL}$ drugs might lead to the development of resistant strains of the parasite. A new control approach is the use of deltamethrin-treated collars, which reduced the risk of infection in dogs by $54 \%$ and in children by $43 \%$ in a study conducted in the Islamic Republic of Iran [16]. Anti-leishmanial vaccines are unfortunately not yet available [17]. Although phlebotomine sand-flies are still susceptible to insecticides, unfortunately the disease quickly re-emerges when the spraying campaigns are discontinued. Therefore early case-finding and treatment of VL is still an essential component of reducing VL mortality and morbidity rates [18].

Our findings showed that mean annual incidence of symptomatic cases of VL decreased significantly from 1.88 per 1000 to 0.77 per 1000 in children $\leq 12$ years old in the periods before and after the intervention respectively, whereas in the control villages, the mean annual incidence of the disease increased significantly from 0.11 per 1000 to 0.23 per 1000 respectively. Our results suggest that establishment of a VL surveillance system can decrease the incidence of $\mathrm{VL}$.

A pilot study to control VL in the focus of Meshkin-Shahr carried out by Nadim et al. in 1991-92 showed that this combined method for control of ZVL (case detection, destruction of stray dogs as well as seopositive ownership dogs, vector control and public health education) was very effective in this area [unpublished report to WHO Special Programme for Research and Training in Tropical Diseases (TDR), project no: 910191]. Although early detection of VL in humans is effective in prevention of death due VL and in reducing the incidence of the disease, it cannot completely eliminate the disease by stopping the transmission cycle. Mohebali et al. showed that $14.2 \%$ ) of domestic dogs in the villages of endemic areas of the Islamic Republic of Iran were positive by DAT [2]. Furthermore, the type of construction of houses in villages and the semi-nomadic life of part of the population play an essential role in the transmission cycle of VL.

As the clinical manifestations of VL have low specificity, confirmatory tests are required to decide which patients should be treated. Such tests should not only be highly sensitive — as VL is a fatal condition - but also need to be highly specific because the current drugs used to treat VL are toxic [12]. DAT has been specifically developed for field use and has been extensively validated in most endemic areas [8,9]. Thirty studies were included in a recent meta-analysis, which gave sensitivity and specificity estimates for DAT of $94.8 \%$ (95\% CI: $92.7 \%-96.4 \%)$ and $85.9 \%$ (95\% CI: $72.3 \%-93.4 \%$ ) respectively [19]. In our study, we used DAT to support the VL surveillance system because it is simpler than many other tests and suitable for field activities. It should be noted that a positive DAT result in endemic areas must be combined with specific clinical manifestations of VL such as mild fever (at least 2 weeks), paleness and anaemia, splenomegaly, hepatomegaly and lymphadenopathy to be considered as VL [5].

We faced some challenges in carrying out routine serological testing due to variations in different batches of DA antigen, the lack of a cold-chain supply for transport of antigen, the need for serial dilutions of blood or serum samples and also the relatively long incubation time (18 hours) for reading the test results. A variant of DAT - the fast agglutination screening test-is very practical under field or rural conditions, and can be used in the future to screen large populations 
of suspected children in a shorter time (2-3 hours) $[20,21]$.

\section{Acknowledgements}

Some parts of this study received financial support from the National Institute of Health Research of the Islamic Republic of Iran, Tehran University of Medical Sciences and also from the Vice-Chancellor for Research, Tehran University of Medical Sciences (Project Nos: 241/74/3, 241/82/54/60, 241/74/81/3/5).
We thank the staff of the DHCs in Meshkin-Shahr and Ardebil as well as all health workers in Meshkin-Shahr villages and the physicians who helped us with this investigation. We thank Dr F. Pourfarzi, Mr D. Emdadi, Mrs N. Modarres, Mr B.Keysan, Dr Arzanloo and Mr M.K Sajadi from Ardebil University of Medical Sciences, Islamic Republic of Iran.

We especially wish to thank $\mathrm{Mr}$ A. Alizadeh, Mrs.N.Mirsamadi, Dr Seif, Dr G. Soleymanzadeh from the East Azerbaijan health centre and $\mathrm{Mr} \mathrm{A}$.
Nasiri, Mrs S. Molaei, Mr I. Moradi, Mrs Z. Rakhshidan, Mrs S. Anvari, Mr A. Malekpour, Mr E. Taghdisi, Mr O. Amohammadi, Mrs S. Charehdar, Dr Gh.R. Khanbaba and our other colleagues in the remote laboratories and fields.

We also thank Dr M. Sedaghat, Assistant Professor of Medical Entomology and Vector Control in the School of Public Health and Institute of Public Health Research, Tehran University of Medical Sciences for editorial comments.

\section{References}

1. Edrissian GhH. Visceral leishmaniasis in Iran and the role of serological tests in diagnosis and epidemiological studies. In: Ozcel MA, Alkan MZ, eds. Parasitology for the 21st century. Wallingford, England, CAB International, 1996.

2. Mohebali $\mathrm{M}$ et al. Epidemiological aspects of canine visceral leishmaniosis in the Islamic Republic of Iran. Veterinary Parasitology, 2005, 129(3-4):243-251.

3. Mohebali M et al. Application of direct agglutination test (DAT) for the diagnosis and seroepidemiological studies of visceral leishmaniasis in Iran. Iranian Journal of Parasitolology, 2006, 1:15-25.

4. Allian DS, Kagan JR. A direct agglutination test for leishmaniasis. American Journal of Tropical Medicine and Hygiene, 1975, 24:232-236.

5. Soleimanzadeh G et al. Epidemiological aspects of kala-azar in Meshkin-Shahr, Iran: human infection. Bulletin of the World Health Organization, 1993, 71(6):759-762.

6. Edrissian $\mathrm{GhH}$ et al. Visceral leishmaniasis: the Iranian experience. Archives of Iranian Medicine, 1998, 1(1):22-26.

7. Veeken $\mathrm{H}$ et al. Comparison of an rK39 dipstick rapid test with direct agglutination test and splenic aspiration for the diagnosis of kala-azar in Sudan, Tropical Medicine and International Health, 2003, 8:164-167.

8. El Harith AE et al. Improvement of a direct agglutination test for field studies of visceral leishmaniasis. Journal of Clinical Microbiology, 1989, 26(7):1321-1325.

9. Zijlstra EE et al. Direct agglutination test for diagnosis and seroepidemiological survey of kala-azar in the Sudan. Transactions of the Royal Society of Tropical Medicine and Hygiene, 1991, 85(4):474-476.

10. Edrissian $\mathrm{GhH}$ et al. Application and evaluation of direct agglutination test in sero-diagnosis of visceral leishmaniasis in man and canine reservoirs. Iranian Journal of Medical Sciences, 1996, 1:119-124.

11. Alvar J et al. Canine leishmaniasis. Advances in parasitology, 2004, 57:1-88.
12. Chappuis F et al. Visceral leishmaniasis: what are the needs for diagnosis, treatment and control? Nature Reviews. Microbiology, 2007, 5:873-882.

13. Ashford DA et al. Studies on control of visceral leishmaniasis: impact of dog control on canine and human visceral leishmaniasis in Jacobina, Bahia, Brazil. American Journal of Tropical Medicine and Hygiene, 1998, 59:53-57.

14. Palatnik-de-Sousa CB et al. Impact of canine control on the epidemiology of canine and human visceral leishmaniasis in Brazil. American Journal of Tropical Medicine and Hygiene, 2001, 65:510-517.

15. Alvar J et al. Canine leishmaniasis: clinical, parasitological and entomological follow-up after chemotherapy. Annals of Tropical Medicine and Parasitology, 1994, 88:371-378.

16. Gavgani AS et al. Effect of insecticide-impregnated dog collars on incidence of zoonotic visceral leishmaniasis in Iranian children: a matched-cluster randomized trial. Lancet, 2002, 360:374-379.

17. Mohebali M et al. Double-blind randomized efficacy field trial of alum precipitated autoclaved Leishmania major vaccine mixed with BCG against canine visceral leishmaniasis in Meshkin-Shahr district, I.R. Iran. Vaccine, 2004, 22:40974100.

18. Desjeux P. Leishmaniasis: current situation and new perspectives. Comparative Immunology, Microbiology and Infectious Diseases, 2004, 27:305-318.

19. Chappuis F et al. A meta-analysis of the diagnostic performance of the direct agglutination test and rK39 dipstick for visceral leishmaniasis. British Medical Journal, 2006, 333:723.

20. Schoone GJ et al. A fast agglutination screening test (FAST) for the detection of anti-Leishmania antibodies. Transactions of the Royal Society of Tropical Medicine and Hygiene, 2001, 95:400-401.

21. Babakhan $L$ et al. Rapid detection of Leishmania infantum infection in dogs: a comparative study using fast agglutination screening test (FAST) and direct agglutination test (DAT) in Iran. Parasitology Research, 2009, 105:717-720. 